Article

\title{
Application of FHCal for Heavy-Ion Collision Centrality Determination in MPD/NICA Experiment
}

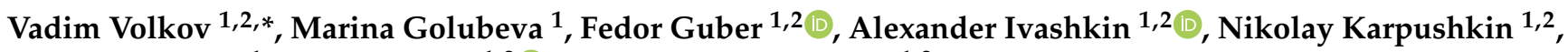 \\ Sergey Morozov ${ }^{1}$, Sultan Musin ${ }^{1,2} \mathbb{D}$ and Alexander Strizhak ${ }^{1,2}$ \\ 1 Institute for Nuclear Research RAS, 117312 Moscow, Russia; marina@inr.ru (M.G.); guber@inr.ru (F.G.); \\ ivashkin@inr.ru (A.I.); nkarpushkin@mail.ru (N.K.); morozovs@inr.ru (S.M.); musin.sa@phystech.edu (S.M.); \\ strijakao@mail.ru (A.S.) \\ 2 Moscow Institute of Physics and Technology, 115184 Moscow, Russia \\ * Correspondence: volkov@inr.ru
}

check for updates

Citation: Volkov, V.; Golubeva, M.; Guber, F.; Ivashkin, A.; Karpushkin, N.; Morozov, S.; Musin, S.; Strizhak, A. Application of FHCal for

Heavy-Ion Collision Centrality Determination in MPD/NICA

Experiment. Particles 2021, 4, 236-240. https://doi.org/10.3390/

particles4020022

Academic Editor: Peter Senger

Received: 30 April 2021

Accepted: 27 May 2021

Published: 31 May 2021

Publisher's Note: MDPI stays neutral with regard to jurisdictional claims in published maps and institutional affiliations.

Copyright: (C) 2021 by the authors. Licensee MDPI, Basel, Switzerland. This article is an open access article distributed under the terms and conditions of the Creative Commons Attribution (CC BY) license (https:/ / creativecommons.org/licenses/by/ $4.0 /)$.

\begin{abstract}
Two approaches related to the centrality determination in heavy-ion Multi-Purpose Detector (MPD) experiments, using charge-particles multiplicity in Time Projection Chamber (TPC) and the energy deposition in Forward Hadron Calorimeter (FHCal) are discussed. The main features of the FHCal are the fine transverse segmentation and the beam holes in the center of the calorimeters. Leaking the heavy non-interacting fragments (spectators) leads to ambiguity in the dependence of energy deposition in the FHCal on the collision centrality. However, the calorimeter transverse segmentation allows one to measure the energy distributions in each of the FHCal modules and to construct combined observables to resolve the problems associated with the beam hole. The comparison of these approaches in the collision centrality measurements is discussed.
\end{abstract}

Keywords: NICA; heavy-ion; centrality; MPD; FHCal

\section{Introduction}

The centrality is the global observable determining the geometry of the nucleusnucleus collision for the event characterization. The measurements of centrality can be performed with participants or spectators of colliding nuclei. The charged-particle multiplicity allows the number of participants to be estimated. Usually, the Glauber Monte Carlo codes are used to evaluate the number of participants from the particle multiplicity in the tracking detectors [1,2]. The number of spectators (non-interacting nucleons in the colliding nuclei) is measured by the forward hadron calorimeters placed near the beam axis. Monte Carlo simulations of the spectator production have been performed with transport models [3].

The MPD experiment at NICA accelerator complex [4] has both options for the determination of the collision centrality. The Multi-Purpose Detector (MPD) [5] will be used to study the heavy-ion collisions at energies of 4-11 AGeV in the center-of-mass frame. The scheme of the MPD experiment is shown in Figure 1, left. The first stage of the experiment setup includes Time Projection Chamber (TPC), Time-of-Flight system (TOF), Electromagnetic Calorimeter (ECal), Forward Hadron Calorimeter (FHCal) and fast Forward Detector (FD). All parts of the setup are placed in a superconducting magnet. The FHCal [6] will measure the collision geometry, namely the centrality and the reaction plane orientation. Two equivalent FHCal parts consist of 44 individual modules (see Figure 1, right) and will be symmetrically placed at the opposite sides of the collision point. The transverse segmentation of the calorimeter makes it possible to measure spatial energy distributions and to construct different observables that combine both spatial and amplitude information in the calorimeter.

The MPD setup allows the determination of the heavy-ion collision centrality in two ways; estimating the number of produced particles from track multiplicity in TPC or 
number of spectators measuring deposited energy in the FHCal. This paper describes centrality determination with the FHCal in details. The comparison with the track multiplicity approach is discussed.
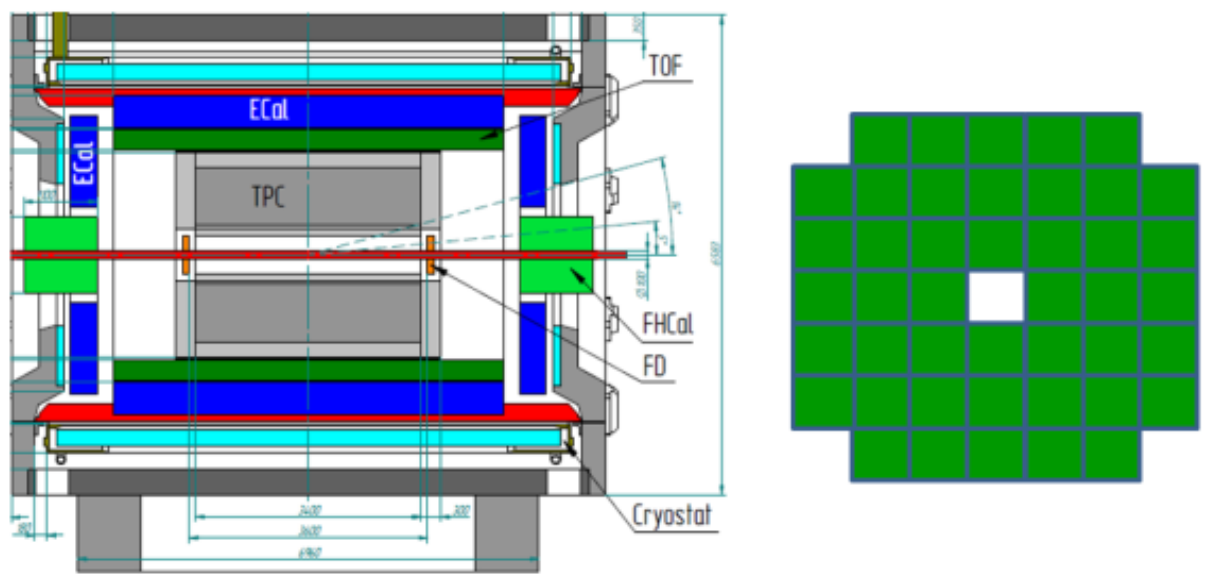

Figure 1. Left: the side view of the MPD setup. Right: schematic view of FHCal transverse segmentation.

\section{Centrality Determination with the FHCal}

The main problem in the centrality measurements with the FHCal is the presence of the central beam hole with transverse sizes of $15 \times 15 \mathrm{~cm}^{2}$. The fragments with small transverse momenta from interacting nuclei leak into this hole. This leakage is especially significant for the peripheral collisions, where the heavy spectator fragments are produced. As a result, the deposited energy in the FHCal is comparable for the central and peripheral events that lead to the ambiguity in the dependence of energy deposition on the collision centrality.

An advanced approach for the centrality measurements with the application of the spatial distributions of the deposited energies in the FHCal has been developed. The simulations were performed using the DCM-SMM Monte Carlo generator [7] for $\mathrm{Au}-\mathrm{Au}$ reaction at the maximum collision energy $\sqrt{ } \mathrm{s}=11 \mathrm{AGeV}$. This generator allows the bound spectators in all collision centrality range to be identified. The spectrum of the medium mass fragments in DCM-SMM generator is rather similar to the experimentally observed spectrum, which indicates the reliability of the fragmentation mechanism used in the model. This is important because energy deposition in the FHCal strongly depends on the number of detected fragments.

Due to the fine FHCal transverse segmentation, one can measure the energy deposition in each module on an event-by-event basis and construct new experimental observables associated with such energy-space distribution, according to the following procedure. Initially, two-dimensional distribution of deposited energies in FHCal modules is constructed (see Figure 2, left). Then, the energies in the modules with the same polar angles are averaged to ensure the reliable fit of the histogram with a symmetric cone. As a result of two-dimensional linear fit, two new observables, the height and the radius of the cone, are obtained. The correlation between the height of the cone (maximum energy) and the deposited energy in the FHCal is shown in Figure 2, middle. The upper branch of this correlation is related to the central events, while the peripheral events belong to the lower branch. The events were divided into 10 centrality classes with an equal number of events, by constructing the perpendiculars to the envelope of the obtained correlation. Figure 2, right, presents the distribution of impact parameters for each $10 \%$ centrality class. The values of impact parameters are monotonically increasing according to the centrality class index. More details on centrality determination with the FHCal can be found in [8]. 

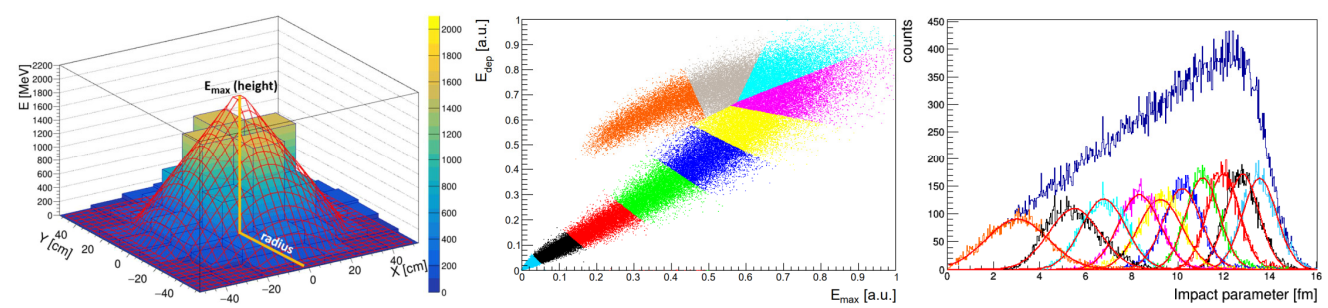

Figure 2. Left: the two-dimensional linear fit of the histogram with energies deposited in each FHCal module. Middle: the correlation between the maximum energy (height of the fit cone) and the deposited energy in the FHCal. Different colors denote 10\% centrality classes. Right: impact parameters distributions for each $10 \%$ centrality class fitted with Gaussian. Colors of the fit lines coincide with the colors of the centrality classes in middle panel.

\section{Comparison of Two Approaches for the Collision Centrality Determination}

The method of collision centrality measurements described above is based on the detection of the spectators in a very forward rapidity region. This method can be compared with another one related to the measurements of the charge-particle multiplicity in the TPC. Figure 3, left, presents the correlation between the energy deposition in the FHCal and the track multiplicity in the TPC. The division to the centrality classes is based on the method described above. There is a good correlation between these observables for the central events. At the same time, the multiplicity is less sensitive to the characterization of the peripheral events.
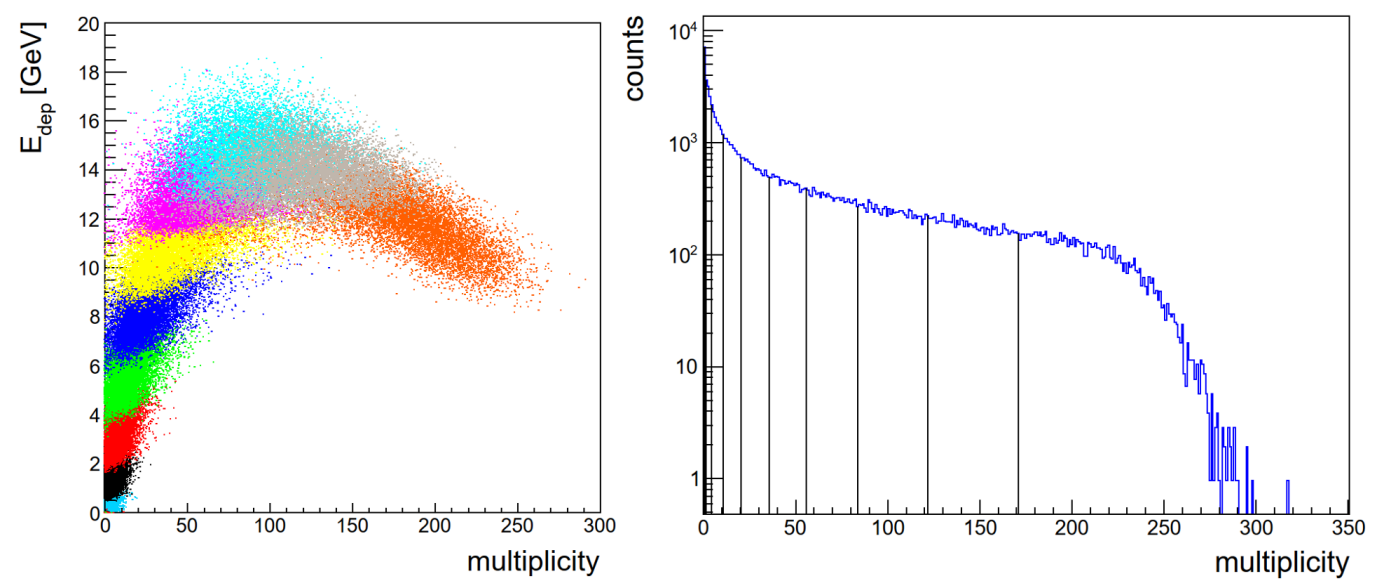

Figure 3. Left: correlation between the energy deposition in FHCal and track multiplicity in TPC. Each color corresponds to $10 \%$ centrality class determined in FHCal (see Figure 2). Right: chargedparticles multiplicity distribution in TPC divided into 10 parts, with $10 \%$ of events in each part.

Quantitatively, the comparison of two approaches for the centrality determination can be done using the number of participants per event in the DCM-SMM generator. For this purpose, the TPC track multiplicity distribution was divided into 10 parts with the equal number of events (see Figure 3, right). Each part denotes the 10\% centrality class. Distributions of participants' number for the TPC and the FHCal centrality classes are shown in Figure 4, left and right, respectively. The events with a larger number of participants are related to the more central events and vice versa.

As seen in Figure 4, the shapes of the distributions in left and right panels are nonidentical, which reflects the selection of different events in the same centrality class.

For each TPC or FHCal centrality class, the mean value of the participants' distribution and its relative standard deviation were calculated and shown in Figure 5. 

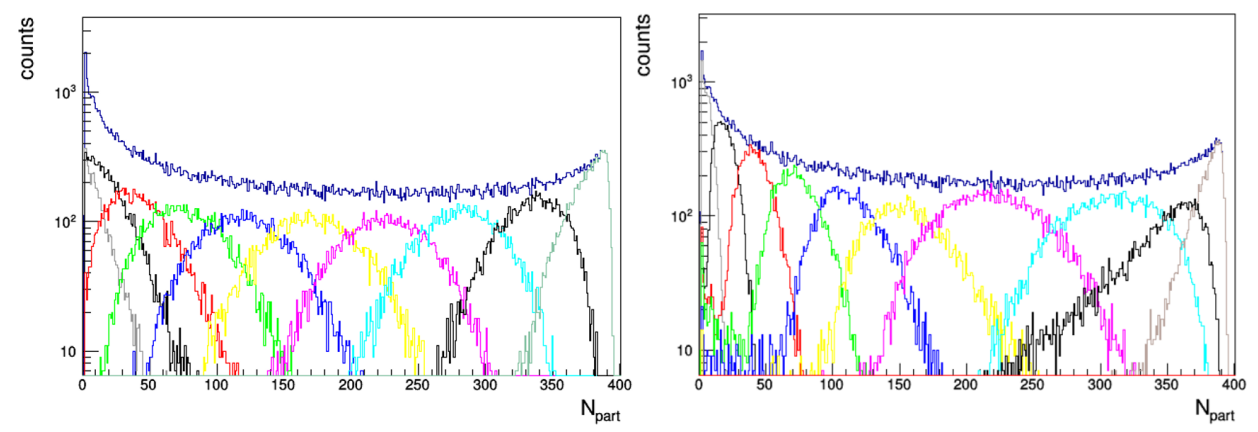

Figure 4. The number of participants' distributions for the centrality classes determined with the TPC (left) and with the FHCal (right). Each color indicate 10\% centrality class determined in FHCal.
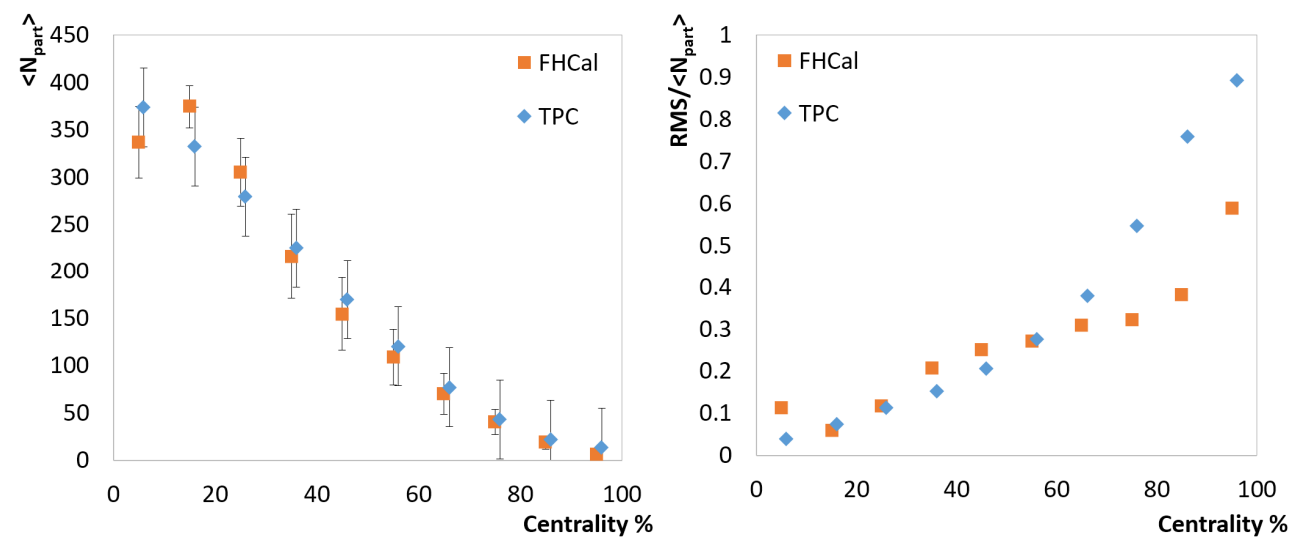

Figure 5. Mean values of the number of participants with error bars that denote the standard deviations of the participants' distributions for the given centrality class (left) and the relative standard deviations of participants' distributions (right) for two methods of centrality determination.

\section{Conclusions}

The MPD/NICA experiment has an ability to measure the collision centrality in both participants' and spectators' regions of the nuclei interactions. Two-dimensional fit of energy depositions in FHCal modules has been proposed and applied to obtain the centrality classes. The FHCal energy deposition and TPC track multiplicity methods of collision centrality determination were compared. The mean values of the participants' number distributions are similar for both cases. The relative widths of the distributions for the central and semi-peripheral classes are comparable in both approaches. For peripheral events, the accuracy of the number of participants is much higher in the case of the FHCal. The presented results confirmed that both approaches are practically equivalent for the selection of the centrality and choice of the method depends on the analysis purposes. For example, the centrality selection by measuring the forward energies in FHCal is of special importance for the study of spectra and multiplicities of the charged particles [9].

Author Contributions: Conceptualization, A.I.; Formal analysis, V.V.; Funding acquisition, A.I.; Investigation, V.V. and A.I.; Methodology, A.I.; Project administration, F.G.; Resources, S.M. (Sergey Morozov); Software, M.G., S.M. (Sergey Morozov), N.K., A.S. and S.M. (Sultan Musin); Supervision, F.G.; Validation, F.G., A.I. and S.M. (Sergey Morozov); Visualization, V.V.; Writing—original draft, V.V.; Writing-review \& editing, V.V. All authors have read and agreed to the published version of the manuscript.

Funding: This research was funded by RFBR, grant number 18-02-40065.

Institutional Review Board Statement: Not applicable.

Informed Consent Statement: Not applicable.

Conflicts of Interest: The author declares no conflict of interest. 


\section{References}

1. Abelev, B.; Adam, D.; Adamová, A.M.; Adare, M.M.; Aggarwal, G.; Aglieri Rinella, M.; Agnello, A.G.; Agocs, A.; Agostinelli, Z.; Ahammed, N.; et al. (ALICE Collaboration). Centrality determination of Pb-Pb collisions at $\sqrt{ } \mathrm{s}_{\mathrm{NN}}=2.76 \mathrm{TeV}$ with ALICE. Phys. Rev. C 2013, 88, 044909. [CrossRef]

2. Adamczewski-Musch, J.; Arnold, O.; Behnke, C.; Belounnas, A.; Belyaev, A.; Berger-Chen, J.C.; Biernat, J.; Blanco, A.; Blume, C.; Böhmer, M.; et al. (HADES Collaboration). Centrality determination of $\mathrm{Au}+\mathrm{Au}$ collisions at $1.23 \mathrm{~A} \mathrm{GeV}$ with HADES. Eur. Phys. J. A 2018, 54, 85. [CrossRef]

3. Buss, O.; Gaitanos, T.; Gallmeister, K.; van Hees, H.; Kaskulov, M.; Lalakulich, O.; Larionov, A.B.; Leitner, T.; Weil, J.; Mosel, U.; et al. Transport-theoretical description of nuclear reactions. Phys. Rept. 2012, 512, 1-124. [CrossRef]

4. NICA—Nuclotron-based Ion Collider fAcility. Available online: http://nica.jinr.ru (accessed on 28 May 2021).

5. Golovatyuk, V.; Kekelidze, V.; Kolesnikov, V.; Rogachevsky, O.; Sorin, A. Multi-Purpose Detector to study heavy-ion collisions at the NICA collider. Nucl. Phys. A 2019, 982, 963-966. [CrossRef]

6. Ivashkin, A.; Finogeev, D.; Golubeva, M.; Guber, F.; Izvestnyy, A.; Karpushkin, N.; Morozov, S. Compact segmented hadron calorimeter for detection of low energy spectators at MPD/NICA facility. Nucl. Instrum. Methods Phys. Res. A 2020, 958, 162240. [CrossRef]

7. Baznat, M.; Botvina, A.; Musulmanbekov, G.; Toneev, V.; Zhezher, V. Monte-Carlo Generator of Heavy-ion Collisions DCM-SMM. Phys. Part. Nuclei Lett. 2020, 17, 303-324. [CrossRef]

8. Volkov, V.; Golubeva, M.; Guber, F.; Ivashkin, A.; Karpushkin, N.; Morozov, S.; Musin, S.; Strizhak, A. Approaches in centrality measurements of heavy-ion collisions with forward calorimeters at MPD/NICA facility. J. Phys. Conf. Ser. 2020, 1690, 012103. [CrossRef]

9. Acharya, A.; Adhikary, H.; Allison, K.K.; Andronov, E.V.; Antićić, T.; Babkin, V.; Baszczyk, M.; Bhosale, S.; Blondel, A.; Bogomilov, M.; et al. (NA61/SHINE Collaboration). Spectra and mean multiplicities of $\pi$ - in central 40Ar+45Sc collisions at 13A, 19A, 30A, 40A, 75A and 150AGeV/c beam momenta measured by the NA61/SHINE spectrometer at the CERN SPS. Eur. Phys. J. C 2021, 81, 397. [CrossRef] 